\title{
Robot Extenics Control Developed by Versatile, Intelligent and Portable Robot Vipro Platform Applied on Firefighting Robots
}

\author{
https://doi.org/10.3991/ijoe.v16i08.15309 \\ L. Vladareanu ${ }^{*}(\varpi)$, V. Vlădareanu, N. Pop, M. Migdalovici \\ Institute of Solid Mechanics of the Romanian Academy, Bucharest, Romania \\ luigiv2007@yahoo.com.sg \\ M. Boșcoianu, S. Pop \\ Transilvania University of Brașov, Brassov, Romania \\ A. Ciocîrlan \\ Institute of Solid Mechanics of the Romanian Academy, Bucharest, Romania
}

\begin{abstract}
The firefighting robot control using intelligent Extenics control interfaces ICEx developed by versatile, intelligent and portable robot VIPRO platform is presented. Subsequently, VIPRO platform architecture, the innovative versatile, intelligent, portable platform, is applied on firefighting robots VIPFiR\&FiTRo, Extenics intelligent control and Universe of Discourse in an Extenics Transformation are detailed. The Extenics Control Method with Fuzzy Smoothing is developed and tested using a simple DC motor configuration in the Matlab / Simulink programming environment. A sequence for the Intelligent Control Interface Code integrated into the VIPRO is presented. The results prove the possibilities for tweaking and optimizing by intelligent Extenics control developed on VIPRO platform in order to obtain improved performance are virtually limitless.
\end{abstract}

Keywords-Intelligent Control; Extenics; Robotics, Real Rime Control; VIPRO Platform.

\section{Introduction}

Extenics is a science whose stated aim is to deal with unsolvable problems, with applications in artificial intelligence, business, marketing, planning, design, control theory and image processing, to name just a few. It is one of the fastest developing new fields of study in the world today. Extenics is a science combining Mathematics, Engineering and Philosophy [1]. With respect to its application and use, it is a trans-disciplinary science, classified as belonging to the wider field of Artificial Intelligence. Extenics Logic extends fuzzy logic, much in the same way that fuzzy logic extends the classical Cantor logic [2]. Cai Wen, in "Extension Set and Non - Compatible Problems", established the foundation of what would later become Extenics Theory [2]. 
The dominant view with respect to the use of Extenics Theory for control applications relates to improving the range of controllability, rather than the quality of control parameters [3-5]. Excellent results have also been obtained by [6, 7].

Extenics was developed by Cai Wen in 1983 and successively, with a major impact in the scientific world of the last few years through results in e-learning, data mining, image recognition, robotics, statistics and management research, among others $[1,8$, 9]. Extension set theory is a mathematical form for representing uncertainty, which is an extension of classic set theory, with applications in many research fields [10-11]. Extenics is a field of study which aims to solve contradictory problems, as is the case of position - force control in the field of robotics, mechatronics and real time control.

\section{Vipro Platform Architecture}

The innovative platform VIP- FiR\&FiTRo (Figure 1) was developed as an open architecture system and adaptive networks integrating Future Internet Systems vision enabling: cyber-physical systems by adaptive networks, intelligent network control systems, human in the loop principles, data mining, big data, and intelligent control interfaces. The network quality of service, shared resources and distributed server network - remote control and e-learning users by interconnected global clouds, were integrated. Based on all the above, the challenges and, therefore, expected progress of VIPFiR\&FiTRo are its ability to be interactive, integrated and competitive with advanced scientific research works.

The technical solution for the innovative versatile, intelligent, portable platform applied on firefighting robots VIP- FiR\&FiTRo includes modules of 3D VIPRO platform designed to work in an open architecture with real time control [10, 12]. Intelligent control interfaces use advanced control strategies based on artificial intelligence algorithms adapted to the type of robot and to the operating environment $[7,11]$. The used types of control are extended control (Extenics), neutrosophic control, and human adaptive control and these control types are implemented using IT\&C real time, rapid processing communications. On the VIPRO platform, the following interfaces were designed: extended control interface, robot neutrosophic control interface and neural nets interface for force-position hybrid dynamic control [6-8, 12].

The concept is that the robotics mobile unit will go to the safe proximity of the firefighting emergency area, in particular fire and rescue operations such as the rescue missions in aircraft/airport, wilderness fire suppression, and search and rescue, including emergency medical services. It can do that as it is equipped with innovative devices that determine the direction and the identification of dangerous clouds and the toxic environment created by combustible materials, their moving direction, nature of agents that contaminate, oxygen deficiency, elevated temperatures, and poisonous atmospheres, provided in safe condition for personnel protection. After the safe stop of the robotics mobile unit, there are the correlated actions of unmanned ground and aerial vehicles (FiR\&FiTRo and UAV), all of these coordinated by the virtual intelligent platform. 


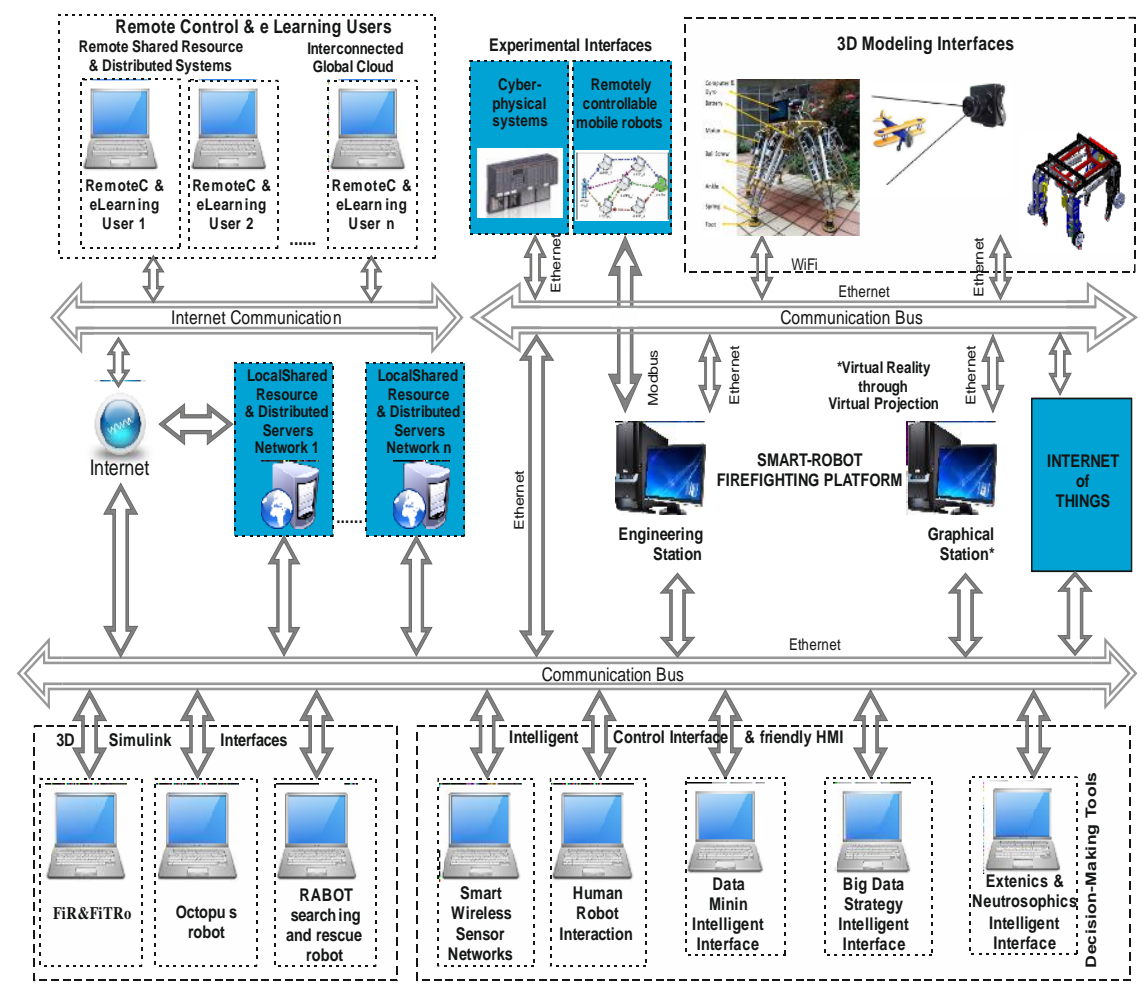

Fig. 1. The VIP- FiR\&FiTRo - Virtual intelligent portable platform of firefighting robot

The need to manage all behaviors and interactions is solved by developing some innovative interfaces for intelligent control based on advanced control strategies, such as extended control (Extenics), neutrosophic control, and human adaptive mechatronics, implemented by high speed processing IT\&C techniques in real time communication for a high amount of data processing, including a remote control and e-learning component and an adaptive networked control. This allow the development of new methodologies, evaluation metrics, test platforms, reproducibility of experiments, and novel approaches to academia-industry co-operation for enabling disruptive product and process innovation and, last but not least, a fire engineering network for research and modeling complex data for quick actions firefighting and management of fire and emergency services.

Robotic control is essential in developing control and perception algorithms for robotics applications. A 3D simulator for mobile robots must correctly control the dynamics of the robots and of the objects in the environment. Moreover, real-time control is important in order to correctly model interactions among the robots and between the robots and the environment, so it is often necessarily an approximation to obtain realtime performance. 


\section{Extenics Intelligent Control}

The aim of the new method described in this work is rather to improve the controller quality, with regard to both such classical metrics as overshoot, settling time and so on, as well as ease of use and reduced complexity. This can be achieved by using concepts from Extenics Theory, such as the Dependent Function, as a means of obtaining a measurement for the incompatibility of the system. The basic idea is using the Dependent Function as a means of judging whether the controlled parameter is within an acceptable range and altering the output of the controller commensurately with the degree of incompatibility shown by the Dependent Function. The degree of compatibility will then determine the scope of the controller action being taken. Thus, it provides a powerful indicator of reduced complexity for the controller status of the system.

Extension set theory is a new set theory which aims to describe the change of the nature of matters, thus taking both qualitative as well as quantitative aspects into account. The theoretical definition for an extension set is as follows: supposing $U$ to be an universe of discourse, $u$ is any one element in $U, k$ is a mapping of $U$ to the real field $I, T U, T k, T u)$ is given transformation, we call:

$$
E(T)=\left\{\left(u, y, y^{\prime}\right) \mid u \in U, y=k(u) \in I, T_{u} u \in T_{U} U, y^{\prime}=T_{k} k\left(T_{u} u\right) \in I\right\}
$$

an extension set on the universe of discourse $U, y=k(u)$ the Dependent Function of $E(T)$, and $y^{\prime}=T_{k} k\left(T_{u} u\right)$ the extension function of $E(T)$, wherein, $T_{U}, T_{k}$ and $T_{u}$ are transformations of the respective universe of discourse $\mathrm{U}$, Dependent Function $\mathrm{k}$ and element $\mathrm{u}$. If $\mathrm{T} \neq \mathrm{e}$, that is to say the transformation is not identical, four more concepts can be outlined, as follows:

positive extensible field (or positive qualitative change field) of $\mathrm{E}(\mathrm{T})$ :

$$
\underbrace{E}_{w_{+}}(T)=\left\{\left(u, y, y^{\prime}\right) \mid u \in U, y=k(u) \leq 0 ; T_{u} u \in T_{U} U, y^{\prime}=T_{k} k\left(T_{u} u\right)>0\right\}
$$

negative extensible field (or negative qualitative change field) of $\mathrm{E}(\mathrm{T})$ :

$$
\underbrace{E}_{w_{-}}(T)=\left\{\left(u, y, y^{\prime}\right) \mid u \in U, y=k(u) \geq 0 ; T_{u} u \in T_{U} U, y^{\prime}=T_{k} k\left(T_{u} u\right)<0\right\}
$$

positive stable field (or positive quantitative change field) of $\mathrm{E}(\mathrm{T})$ :

$$
E_{+}(T)=\left\{\left(u, y, y^{\prime}\right) \mid u \in U, y=k(u)>0 ; T_{u} u \in T_{U} U, y^{\prime}=T_{k} k\left(T_{u} u\right)>0\right\}
$$

negative stable field (or negative quantitative change field) of $\mathrm{E}(\mathrm{T})$ :

$$
E_{-}(T)=\left\{\left(u, y, y^{\prime}\right) \mid u \in U, y=k(u)<0 ; T_{u} u \in T_{U} U, y^{\prime}=T_{k} k\left(T_{u} u\right)<0\right\}
$$

extension boundary of $\mathrm{E}(\mathrm{T})$ :

$$
E_{0}(T)=\left\{\left(u, y, y^{\prime}\right) \mid u \in U, T_{u} u \in T_{U} U, y^{\prime}=T_{k} k\left(T_{u} u\right)=0\right\}
$$

This is further illustrated in Figure 2 [8]. The Extension Set, then, is defined in relation to a transformation and an existing function mapped onto the universe of discourse. 
Following the transformation, the Extension Set is divided into the positive and negative fields with regard to the Dependent Function value. Four subsets are therefore defined: the positive stable, the positive transitive, the negative stable and the negative transitive field. Transilvania University of Braşov Romania.

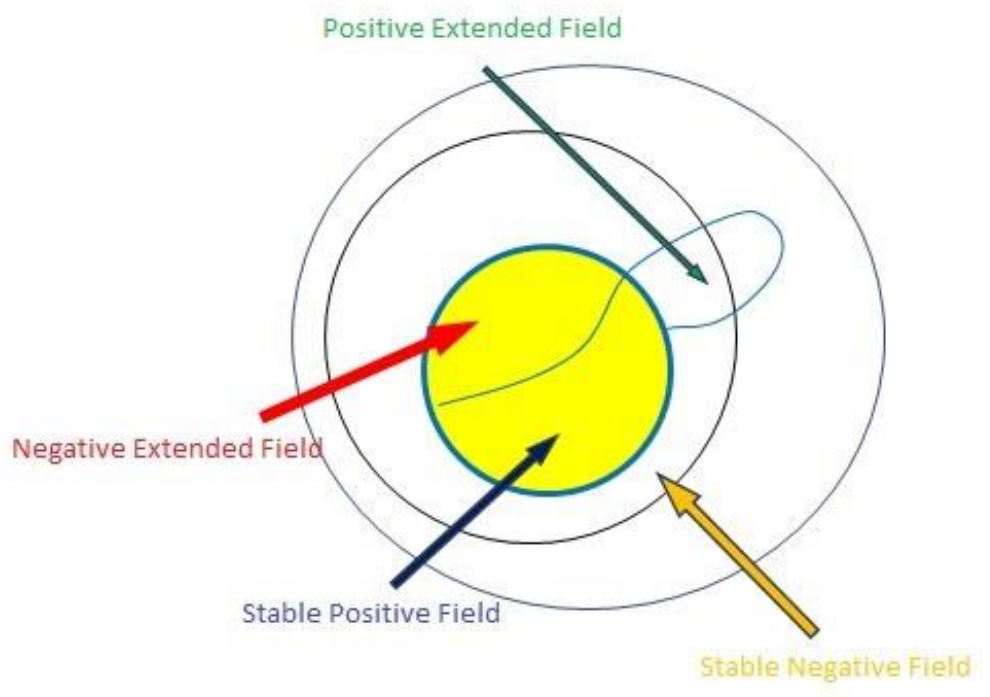

Fig. 2. Universe of Discourse in an Extenics Transformation

The stable fields are those for which the polarity of the dependent function is unaltered by the transformation, whereas transitive (also named extensible) fields are those affected by the change. This provides a useful classification and investigation tool for contradictory problem models. Figure 2 gives another illustration of the four fields of the Extension Set.

The controller classifies the system state into one of four classes and tracks the progress made from incompatibility through to system convergence. The input variable for the fuzzy controller falls within one of four classes, as discussed in the theoretical approach: "Not - Controlled" (NC) for values of the Dependent Function $(k)$ smaller than -1; "Transformable" (Tr) for $k$ between -1 and 0 ; "Acceptable" (Acc) for $k$ between 0 and 1 and "Compatible" (OK) for $k$ greater than 1 . These are the Dependent Function values of the limits of the nested intervals and are the same in any Extenics application, being a property of how the Dependent Function is calculated rather than the problem itself.

Extenics control method with fuzzy smoothing: As mentioned previously, the degree of incompatibility, measured using the Dependent Function, will provide a scale of the output of the controller. The controller simulations and tests are performed using a simple DC motor configuration in the Matlab / Simulink programming environment. The overall representation of the process is in a Simulink. The motor model, while not at all involved, takes into account the existence of load (although this is not used in this 
application) and provides a scope to the armature current, which needs to be monitored, as very high currents may cause permanent damage to a motor in a real-life setting. Within the simulation, this classification is done using the fuzzy controller.

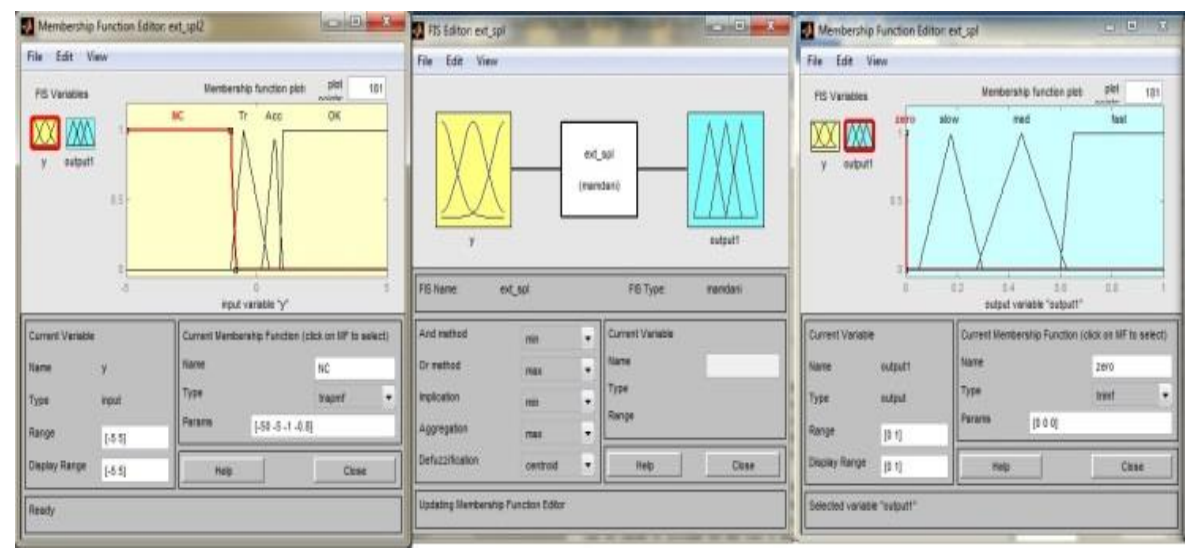

Fig. 3. Fuzzy Controller Sets

Each of the four classes is equated with a set of the input fuzzy linguistic variable. Minor adjustments can be made to account for the way a fuzzy controller processes inputs: the sets do not need to be mutually exclusive and the process is usually smoother when there is some overlapping. The exception to this is in the third set, which does not pass its right outer boundary, so as to prevent the controller from having a residual output once the "Compatible" stage is reached. Figure 3 below shows the arrangements of the input variable and output variable sets.

Considering the three nested interval to be bands around the desired set point value of the process variable, the value of the Dependent Function provides information as to where the current process value is located. Again, the width and position of the bands around the set point value may be altered at will, but for this first implementation they were chosen to be concentric, symmetrical and roughly coinciding with established indicators such as the marks for response time, rise time and settling time. This is explained visually in Figure 4. 


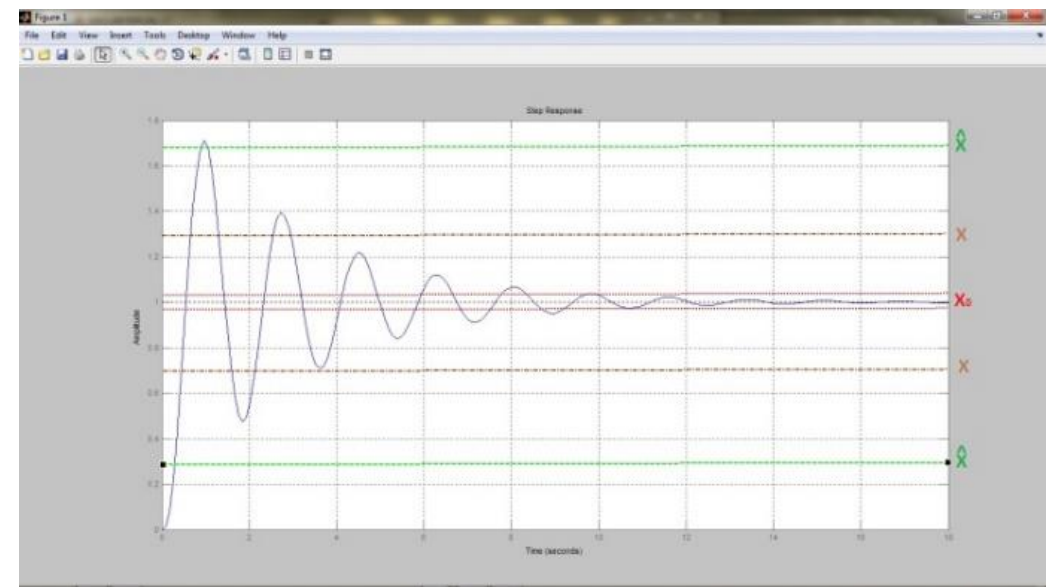

Fig. 4. Nested Intervals of the Extenics Controller

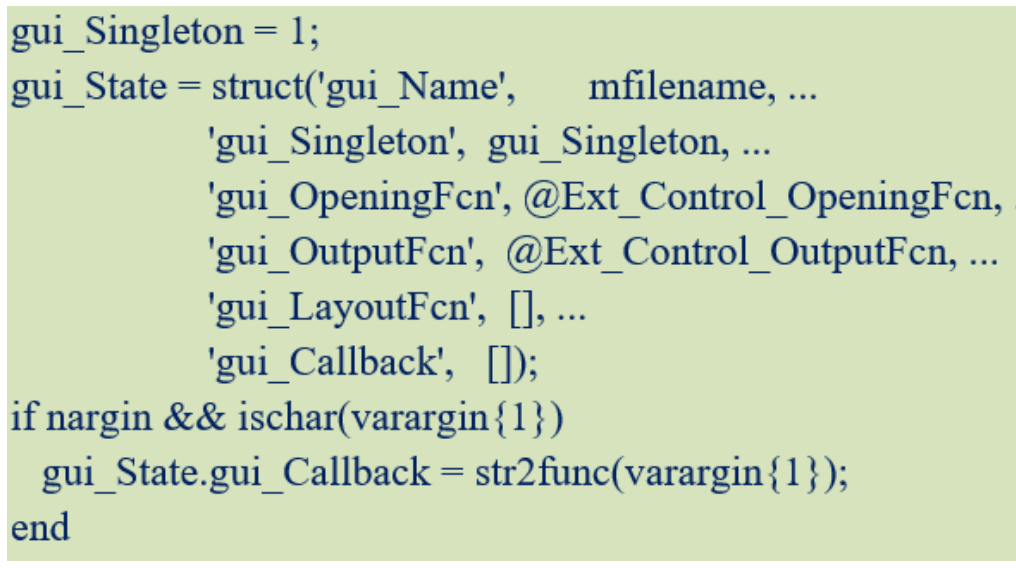

Fig. 5. The Intelligent Control Interface ICEx Code integrated into the VIPRO

The design code of the Intelligent Control Interface for Extended Control Simulation and Validation (ICEx) integrated into the VIPRO platform is presented in Figure 5.

\section{Conclusion}

Development of 3D dynamic perception and visualization, and human-robot interaction software systems are formidably challenging and, accordingly, the activities to support software developments and project management processes are of vital importance to this piece of research. Attribute selected techniques can be categorized on the basis of a number of criteria. Dynamic data come from environmental and wearable sensors, mobile robots and radio communications. 
The ICEx Extenics control benefits greatly in being a novel approach to controller design. The paper proves a working model can be established with basic parameters and the possibilities for tweaking and optimizing in order to obtaining improved performance are virtually limitless. Extenics control is unique in a number of aspects. Perhaps most importantly, it represents a shift in the paradigm of controller structure. While the controllers themselves have evolved greatly over the years, changes in the way one looks at controllers and controller structures have not been frequent.

By way of being an implementation of a more generalized theory, whose aim is precisely to formalize the process of innovation, there is virtually no end to the possibilities for further research. Also, as Extenics Theory continues to grow and mature as a discipline in itself, the theoretical advances made are sure to have a favorable impact on this field of research.

\section{Acknowledgement}

This work was supported by a grant of the Romanian Ministry of Research and Innovation, CCCDI-UEFISCDI, MultiMonD2 project number PN-III-P1-1.2PCCDI2017-0637/33PCCDI/01.03.2018, within PNCDI III, and by the European Commission Marie Sklodowska-Curie SMOOTH project, Smart Robots for Fire-Fighting, H2020-MSCA-RISE-2016-734875 and Yanshan University: "Joint Laboratory of Intelligent Rehabilitation Robot” project, KY201501009, Collaborative research agreement between Yanshan University, China and Romanian Academy by IMSAR, RO. The authors gratefully acknowledge the support of the Robotics and Mechatronics Department, Institute of Solid Mechanics of the Romanian Academy.

\section{References}

[1] Yang Chunyan, Cai Wen, "Extension Engineering", Science Press, Beijing, 2002,

[2] Cai Wen, "Extension Set and Non-Compatible Problems", Advances in Applied Mathematics and Mechanics in China, Peking: International Academic Publishers, 1990, 1-21,

[3] Wang Xingyu, Li Jian. „Extension Control”, Control theory \& applications 1994,11(1):125128 ,

[4] Hu Chen, Wang Xingyu. The Design of Extension Language Controller [C]. Beijing: Matter-element Analysis to Extenics[C]. Beijing: Science and Technology Literature Press, 1995, 12:173-179,

[5] Zhang Yong, Wu Xiaopei, etc. Extension Control Method in a Kind of Multivariable Selfcorrecting System[J]. Journal of Nanjing University of Science and Technology, 2002, 26 (5) : 486-489, 498,

[6] Vladareanu V., Sandru O.I., Schiopu P., Sandru A., Vladareanu L., „Extension Hybrid Force-Position Control of Mechatronics Systems", accepted for publication in Communications in Cybernetics, Systems Science and Engineering, CRC Press, Beijing 2013, https://doi.org/10.1109/icamechs.2013.6681700 
[7] Vladareanu L, Tont G, Ion I, Vladareanu V, Mitroi D, "Modeling and Hybrid Position-Force Control of Walking Modular Robots", ISI Proceedings, Recent Advances in Applied Mathematics, Harvard University, Cambridge, USA, 2010, pg. 510-518, ISBN 978-960-474-1502, ISSN 1790-2769, https://doi.org/10.4316/aece.2010.02026

[8] Vlădăreanu, V., Deng, M., Schiopu, P., "Extenics Controller with Fuzzy Smoothing”, The 2013 International Conference on Advanced Mechatronic Systems, ICAMECHS 2013, https://doi.org/10.1109/icamechs.2013.6681698

[9] O. I. Şandru, L. Vlădăreanu, P. Şchiopu, V. Vlădăreanu, A. Şandru, "Multidimensional Extenics Theory", U.P.B. Sci. Bull., Series A, Vol. 75, Iss. 1, 2013, ISSN 1223-7027, https://doi.org/10.1109/icamechs.2013.6681700

[10] F.Smarandache, L.Vlădăreanu, Applications of Neutrosophic Logic to Robotics - An Introduction, The 2011 IEEE International Conference on Granular Computing Kaohsiung, Taiwan, Nov. 8-10, 2011, pp. 607-612, ISBN 978-1-4577-0370-6, IEEE Catalog Number: CFP11GRC-PRT, https://doi.org/10.1109/grc.2011.6122666

[11] Smarandache Florentin, Vlădăreanu Victor, Applications of Extenics to 2D-Space and 3DSpace, The 6th Conference on Software, Knowledge, Information Management and Applications, Chengdu, China.Sept. 9-11, 2012,

[12] L. Vladareanu, V. Vladareanu, A.I. Gal, O.A. Melinte, European Commission Marie Sklodowska-Curie SMOOTH project, Smart Robots for Fire-Fighting, H2020-MSCARISE-2016-2017-2021734875.

\section{$7 \quad$ Authors}

L. Vladareanu works at Institute of Solid Mechanics of the Romanian Academy, Romania.Email: luigiv2007@yahoo.com.sg

V. Vlădareanu works at Institute of Solid Mechanics of the Romanian Academy, Romania

N. Pop works at Institute of Solid Mechanics of the Romanian Academy, Romania

M. Migdalovici works Institute of Solid Mechanics of the Romanian Academy, Romania

M. Boșcoianu, works at Transilvania University of Braşov Romania

S. Pop works at Transilvania University of Brașov Romania

A. Ciocîrlan works at Institute of Solid Mechanics of the Romanian Academy, Romania

Article submitted 2020-04-29. Resubmitted 2020-05-28. Final acceptance 2020-05-29. Final version published as submitted by the authors. 